\title{
Urban Heat Island in Wuhan and Its Relationship With Nearby Water Bodies
}

\author{
Claire $\mathrm{Xu}^{1}$ \\ ${ }^{1}$ The Experimental High School Attached to Beijing Normal University, China
}

\begin{abstract}
With predictions of global warming to continue into the near future, heat waves are likely to increase both in frequency and severity. Combined with the fast-developing urban areas and sky-rocketing populations in some regions, urban heat island effect becomes increasingly prominent. This trend has caused numerous problems in energy use, human health, and environmental stress. The purpose of the study in this article is to examine the effects of UHI and its impact on nearby water bodies. Through a series of data, which is collected by using Geospatial visualization tool, the study analyzes the extent to which UHI raises the water temperature in Wuhan, China, and compares lakes in different region of Wuhan to explore the impact of modified land surface and human activities. Given the exacerbation of the urban climatic crisis, the study also presents several potential solutions to a sustainable future in urban areas.
\end{abstract}

\section{Introduction of urban heat island}

With rapid urbanization and population growth, the scale of cities and urban landscapes have been greatly enlarged in the past few decades. Urban areas tend to have higher air temperatures than their rural surroundings as a result of gradual surface modifications, which include replacing the natural vegetation with buildings and roads (Hashem, 2005). The term "Urban Heat Island," or UHI, describes this phenomenon.

There are mainly five factors that contribute to the formation of the UHI. First of all, as urban vegetation gets removed and replaced by impervious surface, the evaporation rate is decreased. As these changes take place, the stability of the atmosphere over the city increases, making vertical convection less likely to occur. Thus the temperature near land surface increases. In addition, the concrete, asphalt road and various walls made of artificial materials alter the property of the underlying surface, contributing to the heterogeneity of urban surface. The specific heat capacity of urban areas is therefore smaller than that of rural areas, whose surface property is not altered. Thereby, the urban surface temperature is more likely to increase during daytime and decrease during nighttime. Thirdly, the albedo of urban surfaces material is smaller than that of rural areas, resulting in more absorption and less emission of heat. Moreover, there are many sources of waste heat emission in an urban city, including boilers, heaters, and vehicles in urban cities. These machines and human activities consume much energy, most of which is transmitted into the atmosphere as waste heat. The combustion of fuels also contributes to waste heat emission. Last but not least, the dense architectural complex, roads, and bridges form a coarse surface that lowers the wind speed, decreasing the chance of ventilation and increasing the temperature. All of these factors lead to a higher temperature in urban areas. Since the surrounding rural area has a lower average annual temperature, the city is compared to an "island."

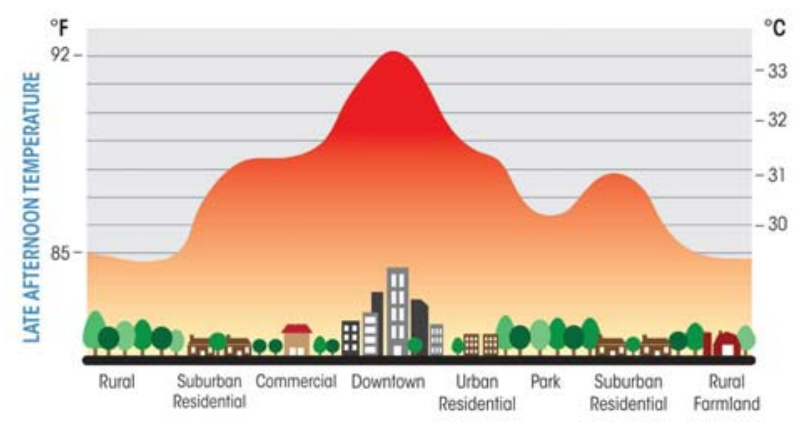

Figure 1. Temperature Pattern of City and Suburb

The hazards of UHI are numerous, and UHI has become one of the largest problems associated with the urbanization and industrialization of human civilization, as the increased temperatures associated with the UHI tend to exacerbate the threats to human health posed by thermal stress. For humans, the concentrated heat will pose various health risks. Pollutants gather in the center of a heat island and achieve a massive increase in concentration, directly irritating people's respiratory mucosa and causing respiratory diseases. The heat island and its side effects can also lead to physical and mental discomfort and sickness, including hyperthermia, vomiting, depression, etc.

$\mathrm{UHI}$ is also a dangerous threat to the environment. The temperature of building surface is much higher than its surroundings. When the rainwater flows from these "hot building," its temperature increases. This warm

*Corresponding author: clairexu116@gmail.com 
water flow then enters the nearby streams, rivers, lakes, and other water bodies, increasing the temperature and creating a thermal pollution that can impact local aquatic lives negatively. The purpose of the study in this article is to examine the effects of UHI and its impact on nearby water bodies. Based on the information above, the main hypothesis of this study is that the water body temperature of urban areas is higher than that of rural areas. In order to prove this hypothesis, the study is carried out by using earth satellites to investigate layers such as land surface temperature, distribution of impervious surfaces, vegetation density, and greenness.

\section{Data Sets and Tools}

The following tools and data sets are used in this research:

1.NASA Worldview: This app from NASA's EOSDIS provides the capability to interactively browse over 900 global, full-resolution satellite imagery layers and then download the underlying data. The most frequently used tool in this study MODIS refers to Aqua Moderate Resolution Imaging Spectroradiometer.

The following layers are used:

- Land Surface Temperature (Aqua/MODIS): The MODIS Land Surface Temperature layer shows the temperature of the land surface in Kelvin $(\mathrm{K})$. The sensor and imagery resolution is $1 \mathrm{~km}$, and the temporal resolution is daily.

- Enhanced Vegetation Index (Aqua/MODIS): Vegetation Indices (MYD13Q1) Version 6 data are generated every 16 days at 250 meter (m) spatial resolution as a Level 3 product.

- Global Man-made Impervious Surface (GMIS): It consists of global estimates of fractional impervious cover derived from the Global Land Survey (GLS) Landsat dataset for the target year 2010. The layer shows the global percent of impervious cover at $30 \mathrm{~m}$ spatial resolution.

- Land/Water Mask (Terra/MODIS): The Terra Moderate Resolution Imaging Spectroradiometer (MODIS) Land Water Mask (MOD44W) Version 6 data product provides a global map of surface water at 250 meters (m) spatial resolution.

2.NASA AppEEARS Earth data: The NASA Application for Extracting and Exploring Analysis Ready Samples (A $\rho$ EEARS) is a web-based tool to search, retrieve, and analyze information derived from Earth Observing (EO) satellites. The following data products were used to extract land/water surface temperature for Wuhan city and nearby rural areas: MODIS LST day and night data at $1 \mathrm{Km} 2$ pixel resolution (MYD11A2_LST_006_Day_1km, MYD11A2_LST_006_Night_1km) for September 6 th of years 2003 to 2015 . This data set was used for a time series analysis of day and night temperatures for selected urban/rural lakes and land areas (polygons). Results are shown and discussed in a later section (Data Analysis).

\section{Overview of Wuhan}

Wuhan, one of the largest cities in Central China, has an intense UHI effect. The city of Wuhan is surrounded by mountains on three sides and only opens on one side. It is often controlled by subtropical highs every summer because of its location at the middle reaches of the Yangtze River. The airflow in Wuhan sinks and warms, the cloud cover is sparse, and the radiation is strong. Occasionally, there are several periods of sunny and hot weather (China meteorological administration). In addition, there are many rivers and lakes in the city, so the evaporated water will stay in the large cloud cluster, leading to excessive heat covering the entire city. Figure 2 and 3 show how the man-made impervious surface coincides with the intensity of night lights. Together, these figures indicate the major urban area of Wuhan. As Wuhan experiences rapid urbanization and population growth, the problem of UHI gradually emerges. The city population ranking of 2019 in China indicates that Wuhan now occupies the 8th place in total population, and its population, unlike many other cities, keeps on growing. The consequence of it would be an increasing burden on local environment. As a result, there has already been some investigations of the possibility of building "smart cities" to alleviate the climatic impact of Wuhan's urban area, and government is trying to solve the environmental crisis that comes along with the urban sprawl. To analyze current situation and address some of the problems related to UHI, this study focuses on Wuhan as the research object.

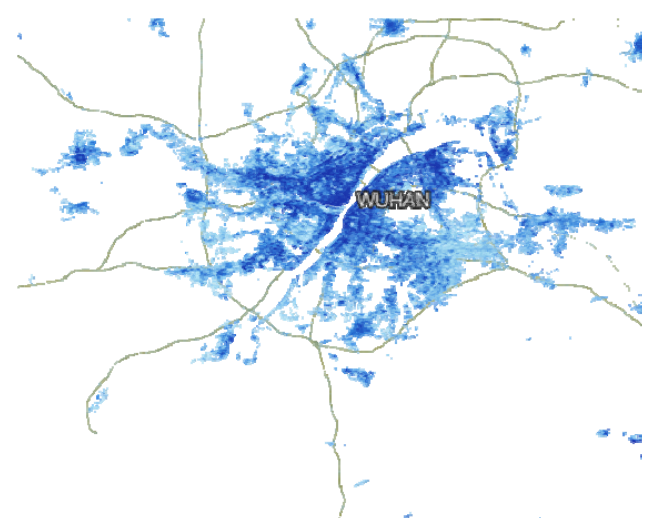

Figure 2. Wuhan Impervious Surface.

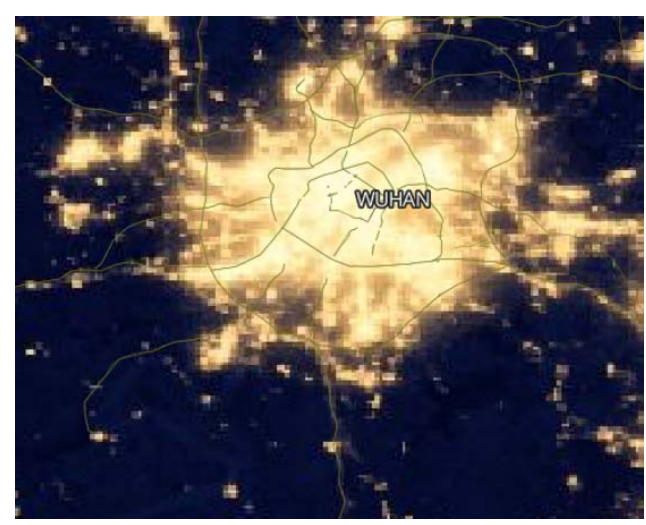

Figure 3. Wuhan At Night 


\section{Data Analysis}

The difference between an urban and rural lake is the distance of them to the impervious surface. Urban lakes are within five kilometers to impervious surface, whereas rural lakes are at least five kilometers away from impervious surface. To verify the pattern of water body temperature changes, lakes with similar latitude (within 100 kilometers) and altitude are chosen to avoid potential influences from other factors. For example, lower latitude and altitude may lead to higher water temperatures. The measurement aims at the time period between year 2003 and 2015. The data is extracted on September 6th each year. The land surface temperature is measured simultaneously near the lakes. The NASA Worldview, with LST layer and impervious surface layer on, shows the following pattern:

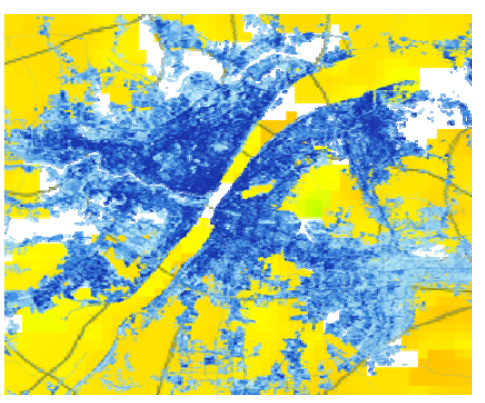

Figure 4. 2003.9.6

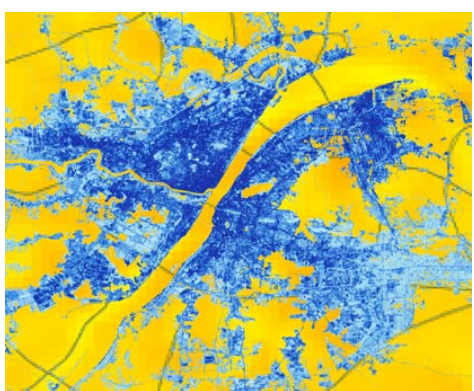

Figure 5. 2009.9.6

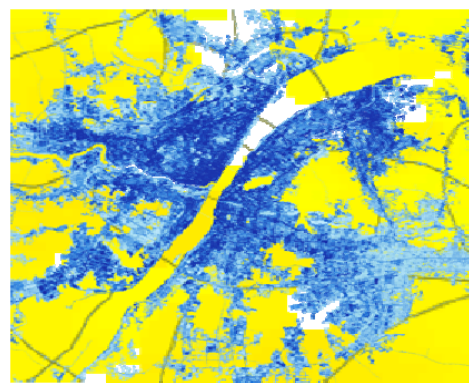

Figure 6. 2015.9.6

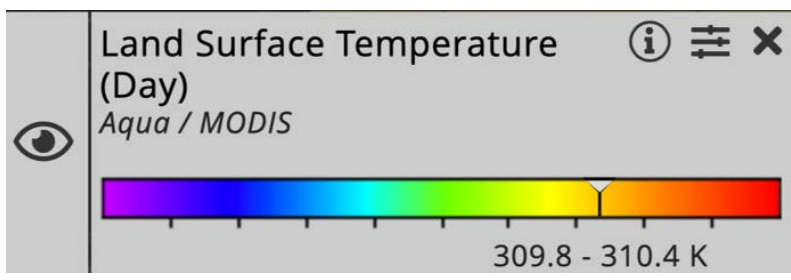

Figure 7. Color Palette of LST (NASA Worldview)
The color on the color palette changes as the temperature changes, with warmer color indicating higher temperature. Therefore, the color orange of the given image indicates a comparably high temperature. It is obvious that an exceptionally higher temperature occurred in year 2009. This may be due to the trend of global warming and increasingly intensive human activity, one of which is urban sprawl, resulting in a significant increase in land surface temperature. This trend sets the basis of an UHI. However, due to the policies of local government to alleviate the intensifying heat, the land surface temperature in year 2015 decreases slightly and shows a lower degree compared to that of year 2009 .

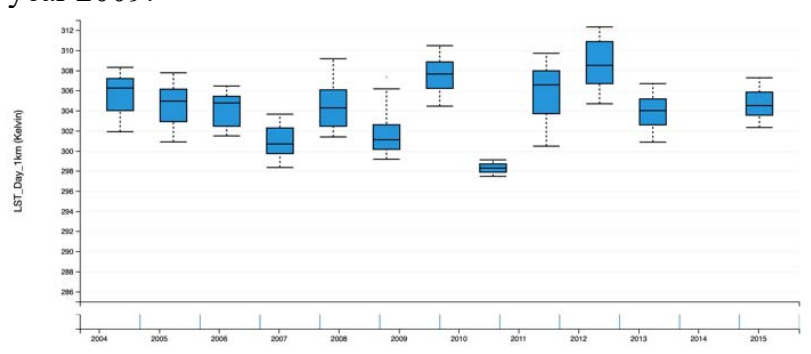

Figure 8. Urban Land Day

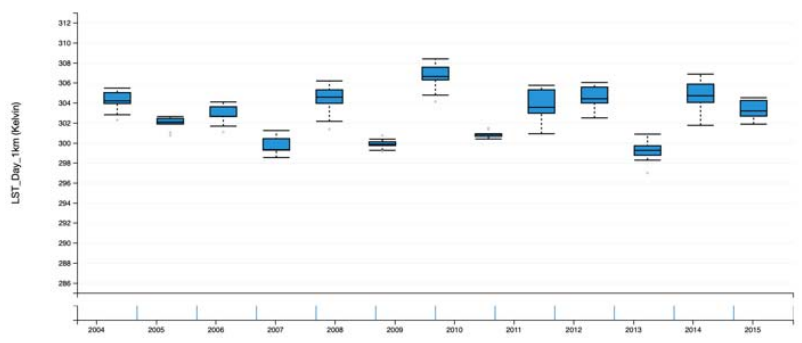

Figure 9. Rural Land Day

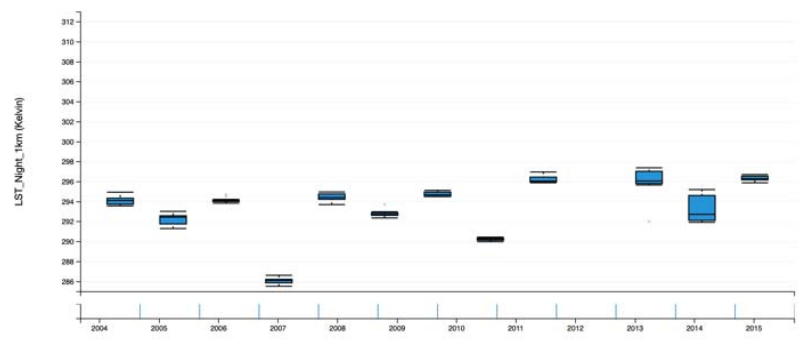

Figure 10. Urban Land Night

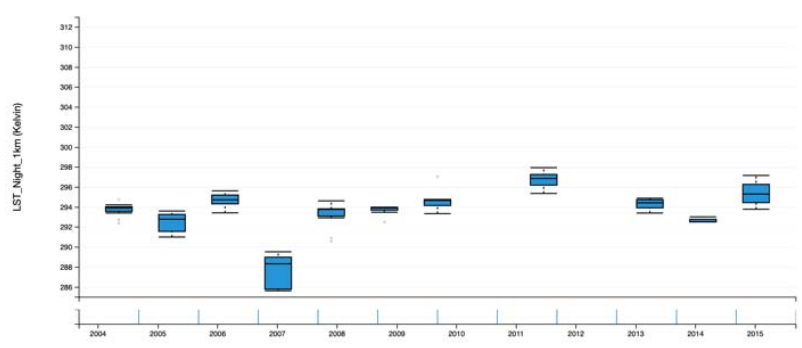

Figure 11. Rural Land Night

As shown by the box plot data from NASA above, land surface temperatures of urban areas during daytime are higher than those of rural areas. The mean values for daytime rural LST in year 2003, 2009, and 2015 are 
$304.2 \mathrm{~K}, 306.6 \mathrm{~K}$, and $303.3 \mathrm{~K}$. The corresponding mean values for daytime urban LST are $305.4 \mathrm{~K}, 307.4 \mathrm{~K}$, and $304.7 \mathrm{~K}$. These data tell us that in general all of the daytime temperatures in urban areas are higher. In fact, every year between year 2003 and year 2015 follows this same pattern. For nighttime LST, the mean values for rural area in year 2003, 2009, and 2015 are 293.7K, 294.6L, and $295.4 \mathrm{~K}$. The corresponding mean values for nighttime urban LST are 294.1K, 294.8K, and 296.3K. It is clear that, whether daytime or nighttime, the land surface temperatures of urban areas are always higher than those of rural areas. This also indicates the actual existence of UHI. If the nighttime temperature is subtracted from the daytime temperature, the differences of day-night LST in urban area in year 2003, 2009, and 2015 are $11.3 \mathrm{~K}, 12.6 \mathrm{~K}$, and $8.4 \mathrm{~K}$. And the corresponding values in rural areas are $10.5 \mathrm{~K}, 12 \mathrm{~K}$, and $7.9 \mathrm{~K}$. It is thus derived that the temperature difference between daytime and nighttime is smaller in rural areas. This can be explained by the altered land surface with lower specific heat capacity in cities, experiencing a more significant increase in temperature when absorbing the same amount of heat.

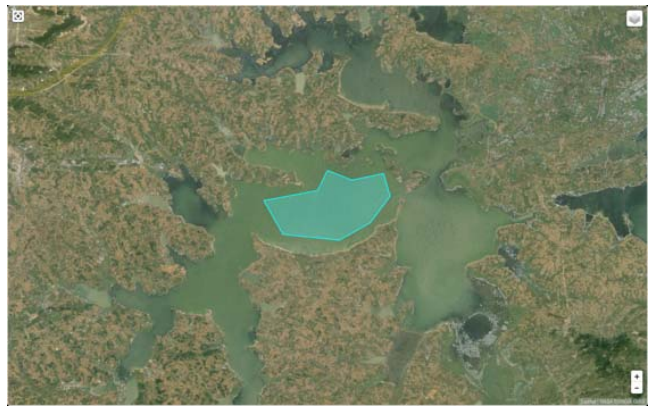

Figure 12. Rural Lake Sample

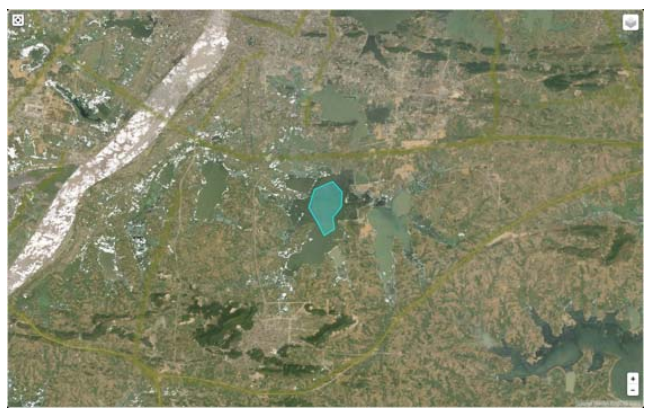

Figure 13. Urban Lake Sample

To find out whether the urban water bodies experienced the same pattern of temperature increase, a set of data is extracted and shown below:

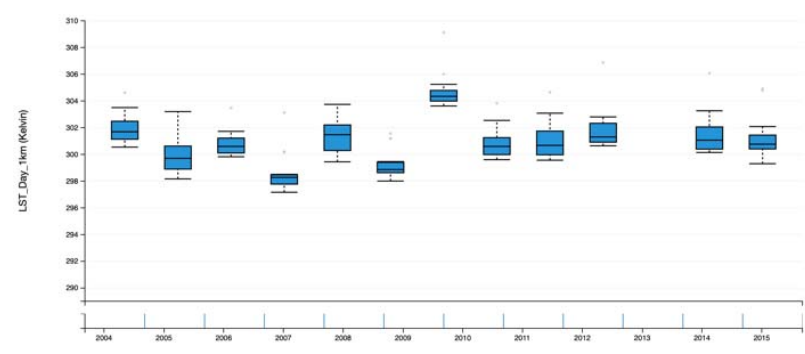

Figure 14. Urban Lake Day

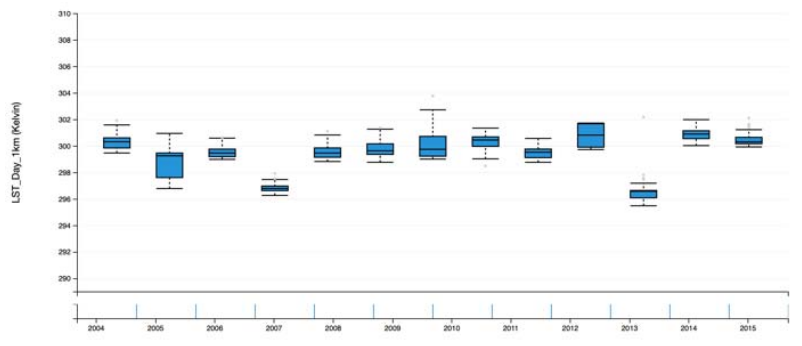

Figure 15. Rural Lake Day

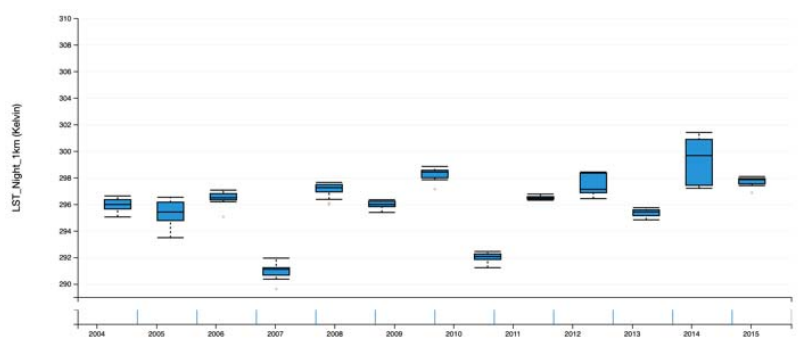

Figure 16. Urban Lake Night

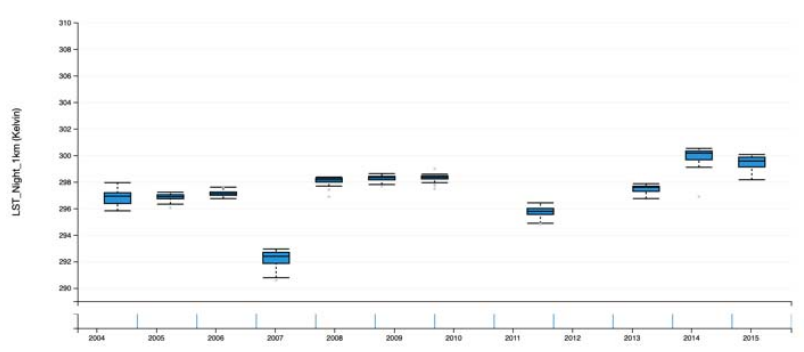

Figure 17. Rural Lake Night

The measurement of lake surface temperature is of the same time period as the measurement of land surface temperature. The data of the designated three years for daytime rural areas are $300.4 \mathrm{~K}, 300.2 \mathrm{~K}$, and $300.5 \mathrm{~K}$, whereas the corresponding urban data are $301.9 \mathrm{~K}$, $304.7 \mathrm{~K}$, and $301.3 \mathrm{~K}$. The same set of data for nighttime rural areas are $296.9 \mathrm{~K}, 298.31 \mathrm{~K}$, and $299.5 \mathrm{~K}$, whereas the corresponding urban data are $296.0 \mathrm{~K}, 298.30 \mathrm{~K}$, and 297.7K (The temperatures for 2009.9.6 keep two decimal places for comparison). There is a trend that during the day, urban lakes have a higher temperature than rural lakes; during the night, urban lakes have a lower temperature than rural lakes. Therefore, this indicates that the temperatures in urban lakes vary more significantly than those of rural lakes. According to the formula of thermodynamics:

\section{$\mathrm{Q}=\mathrm{c} * \mathrm{~m} * \Delta \mathrm{T}$}

(Q-total amount of heat; c-specific heat capacity; m-Mass; $\Delta \mathrm{T}$ temperature change)

The $\Delta \mathrm{T}$ is larger in urban areas. From the satellite picture above, the selected lake sample in urban areas is smaller than its rural counterpart. However, the darker color in the urban lake indicates that the water is deeper than the light-colored rural lake. Therefore, the impact of mass can be ignored. From the formula, the amount of heat absorbed by urban lakes is larger compared with rural lakes. On the positive side, the urban water body helps regulate heat intensity by absorbing much latent heat in the atmosphere. But the potential risk to the water 
body and the aquatic lives is considerable. The varying water temperatures in urban areas may affect the concentration of dissolved oxygen, rendering it too high or low, thus threatening the aquatic lives.

The higher water temperature in urban areas is caused by several different factors. Firstly, the impervious surface in cities prevents the rainwater from infiltrating the soil, as shown in figure 18. Thus the water flows on the land surface and absorbs heat brought about by the UHI effect, eventually flowing into the nearby water body and increasing its temperature. Secondly, the vegetation damage caused by urban sprawl can lead to warm water runoff. The roots of the vegetation are able to maintain soil and water. Therefore, the absence of such maintenance in urban areas will cause soil erosion and water runoff. The vegetation index near the selected rural lake is 0.410-0.418. By contrast, the vegetation index near the selected urban lake is $0.060-0.064$, as shown in figure 19. Higher vegetation index means more intense vegetation coverage, as shown in the color palette in figure 20 . Consequently, there is a marked runoff of water, which is slightly warmer in urban areas. The circled two areas below are the two lakes investigated. The correlation is clear that proximity to the impervious surfaces as well as lower vegetation index both serve as proof to the existence of UHI effect, bringing about highertemperature water bodies.

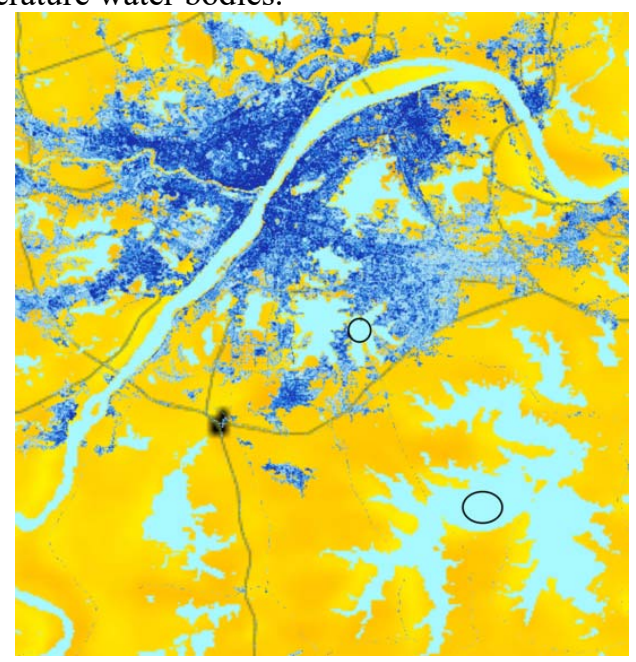

Figure 18. Proximity to Impervious Surfaces

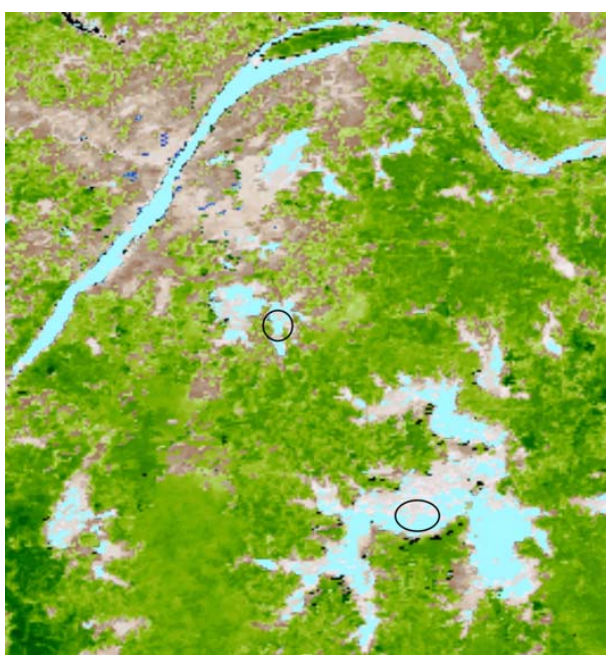

Figure 19. Vegetation Index

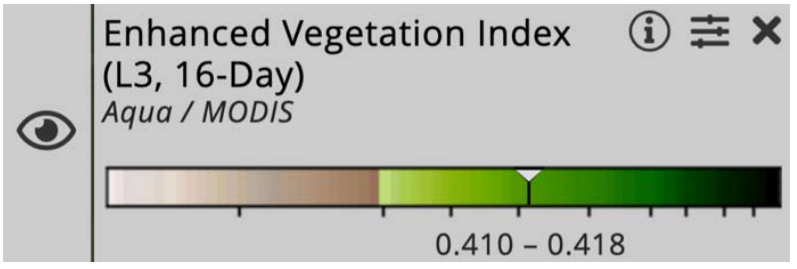

Figure 20. Color Palette of Enhanced Vegetation Index (NASA Worldview)

\section{Prevention Measures}

Increase of the ambient air temperature in cities caused by the urban heat island phenomenon has a serious impact on the economic and social system of cities. (Akbari \&. Cartalis, 2016) To alleviate the long-lasting effect of UHI, the proper designing of the city is critical. Increasing the greenbelt and water area in cities can reduce the accumulating heat in urban areas through more evaporation and heat absorption. Since Wuhan has a complex water system, rain garden can be implemented in urban areas, adding to the greenness of the city so that district cooling can be achieved.

Increasing artificial water evaporation replenishment, such as fountains, sprays, and water mist irrigation can also reduce atmospheric temperature. Additionally, the control of building and population density is also essential to UHI prevention, due to the reason that populated areas are often high energy-consumption areas and potential high temperature zones. One measure is to keep only the central government in center city and reallocate the remaining institutions to satellite cities and connect them to center city by subway or other urban transportation establishments.

For architecture planning, the buildings can try to utilize light color surface to increase the heat albedo. Permeable roads can be adopted to store rainwater and decrease land surface temperature (EPA, Heat Island Cooling Strategies). Among all of these practical solutions, it's better to combine some of them and carry out a comprehensive plan to deal with the urban climatic crisis. 


\section{Conclusion}

Based on the data collected by NASA Worldview and NASA AppEEARS, it can be testified that the UHI effect in Wuhan poses a risk on the urban water body by increasing the water temperature abnormally. Both human and the surrounding environment are negatively impacted by the increasingly high temperature. Though the high specific heat of water can alleviate the effect of UHI, the detrimental impact still exists and will continue to exacerbate human society if UHI isn't dealt with immediately. Hopefully, several measures of city planning can be implemented to improve the situation. This requires collaboration between policymakers, practitioners, and researchers in the future. Meanwhile, the challenge left for the future would be the design of a new set of infrastructure that can prevent UHI from happening in the first place and a well-developed system of policies that ensure the human activities in the cities don't interfere too much with the environment. Above all, decision makers should keep the awareness of sustainable development to minimize environmental impact when constructing new urban landscapes.

\section{References}

1. Akbari, Hashem. Energy Saving Potentials and Air Quality Benefits of Urban Heat Island Mitigation. United States: N. p., 2005. Web. https://www.osti.gov/servlets/purl/860475c

2. China meteorological administration, www.cma.gov.cn (accessed in January 2020)

3. Hashem Akbari, Constantinos Cartalis, Denia Kolokotsa, Alberto Muscio, Anna Laura Pisello, Federico Rossi, Matheos Santamouris, Afroditi Synnefa, Nyuk Hien Wong \& Michele Zinzi (2016) Local climate change and urban heat island mitigation techniques - the state of the art, Journal of Civil Engineering and Management, 22:1, 1-16, DOI: $10.3846 / 13923730.2015 .1111934$.

file:///Users/ClaireXu/Downloads/2312Article\%20Text-5012-1-10-20180606.pdf

4. Heaviside, C., Macintyre, H. \& Vardoulakis, S. The Urban Heat Island: Implications for Health in a Changing Environment. Curr Envir Health Rpt 4, 296-305 (2017). https://doi.org/10.1007/s40572017-0150-3

5. NASA Worldview, https://go.nasa.gov/38R98il (accessed in January 2020)

6. NASA

AppEEARS, https://lpdaacsvc.cr.usgs.gov/appeears/ (accessed in January 2020)

7. Tan, J., Zheng, Y., Tang, X. et al. The urban heat island and its impact on heat waves and human health in Shanghai. Int J Biometeorol 54, 75-84 (2010). https://doi.org/10.1007/s00484-009-0256-x

8. Urban Heat Island, National Geographic, https://www.nationalgeographic.org/ency clopedia/urban-heat-island/ (accessed in January 2020)
9. United States Environmental Protection Agency(EPA), Heat Island Cooling Strate gies, https://www.epa.gov/heat-islands/heat-islandcooling-strategies (accessed in January 2020) 